\title{
Review
}

Neuro[mmunoModulation

\section{Intracellular Pathogen Infections and Immune Response in Autism}

\author{
Renata Torres Abiba, Alexandru Gaman ${ }^{c, d}$ Aroldo A. Dargéele,f \\ Ryad Tamouza d, g, h Flávio Kapczinskig, i,j Carmem Gottfried ${ }^{b, k, l}$ \\ Marion Leboyer ${ }^{c, d}$
}

${ }^{a}$ Department of Nutrition, Federal University of Pelotas (UFPel), Pelotas, Brazil; ${ }^{\text {b }}$ Translational Research Group in Autism Spectrum Disorder (GETTEA), Clinical Hospital of Porto Alegre, Porto Alegre, Brazil; ${ }^{\top}$ Translational Psychiatry Laboratory, INSERM U955, AP-HP, DHU PePSY, Pôle de Psychiatrie des Hôpitaux Universitaires Henri Mondor, Université Paris-Est-Créteil, Paris, France; ${ }^{~}$ Fondation FondaMental, Fondation de Coopération Scientifique, Créteil, France; ${ }^{\mathrm{e}}$ Institut Pasteur, Unité Perception et Mémoire, Paris, France; ${ }^{\mathrm{f} C e n t r e ~ N a t i o n a l ~ d e ~ l a ~ R e c h e r c h e ~ S c i e n t i f i q u e, ~}$ Unité Mixte de Recherche 3571, Paris, France; 9INSERM, U1160, Hôpital Saint Louis, Paris, France; h Université Paris Diderot, Sorbonne Paris-Cité, Paris, France; iNational Institute for Translational Medicine, Hospital de Clínicas de Porto Alegre, Porto Alegre, Brazil; ' Molecular Psychiatry Laboratory, Graduate Program in Medicine, Department of Psychiatry, Federal University of Rio Grande do Sul, Porto Alegre, Brazil; k Department of Biochemistry, Federal University of Rio Grande do Sul (UFRGS), Porto Alegre, Brazil; 'National Institute of Science and Technology on Neuroimmunomodulation-INCT-NIM, Oswaldo Cruz Foundation, Rio de Janeiro, Brazil

\section{Keywords}

Autism spectrum disorder · Pathogens · Infection . Inflammation

\begin{abstract}
Background/Aims: Perinatal exposure to infections during critical developmental periods is a promising area of study in autism spectrum disorder (ASD). Epidemiological data has highlighted this relationship, pointing out significant correlations between perinatal exposure to pathogens and the occurrence of ASD. The aim of this review is to critically examine the present state of the art on intracellular pathogenic infection during pregnancy and postnatally, pointing out possible correlations with the development of ASD. Meth-
\end{abstract}

\section{KARGER}

(c) 2018 S. Karger AG, Basel

E-Mail karger@karger.com

www.karger.com/nim ods: We reviewed and collected studies concerning potential associations between intracellular pathogens like viral, bacterial, and parasite infection and the risk of ASD. Results: We included 14 publications, considering bacterial and/or viral infection that demonstrated the potential to trigger ASD. Nine case-control studies were included and 5 of them reported an association between infections and ASD. One of the 2 cohorts investigated demonstrated that maternal infection increased the risk of ASD in the offspring. Three crosssectional studies demonstrated that ASD patients presented with chronic infections and active neuroinflammatory processes. Most of the reports suggest inflammatory response as a common factor, and interleukin 6 appears to be a keyplayer in this process. Conclusion: The immune responses generated by organisms that cause perinatal maternal infec- 
tion, i.e., bacteria, viruses, or parasites, have been associated with the development of autism in offspring. Physiological changes transmitted from the mother during chronic or acute inflammation should be further investigated so that modulatory preventive measures can be developed.

(c) 2018 S. Karger AG, Basel

\section{Introduction}

Autism spectrum disorder (ASD) is a group of heterogeneous, neurodevelopmental, pathological conditions characterized by deficits in social interactions and repetitive patterns of behaviors and interests [1]. The number of reported ASD cases has recently increased to reach the alarming level of 1 in 68 children in the USA, with a 50fold increase in reported cases between 1975 and 2012 [2].

The precise etiology of ASD remains unknown, albeit a contribution of several genetic and environmental factors is now acknowledged [3]. Accordingly, potential important contributors to ASD risk may include an underlying immune dysfunction reflected by a proinflammatory status, possibly induced by maternal infections during the perinatal period [4]. Interestingly, this condition has been observed repeatedly, together with interindividual immunogenetically driven susceptibility such as human leukocyte antigen (HLA) genes/haplotypes and genetic variants that disrupt MET transcription [5-7]. In line with this, altered cellular immunity and an altered cytotoxic function of natural killer (NK) cells have been reported in subjects with ASD (aged 16-18 years); both possibly confer broadly inefficient anti-infectious responses against potential ASD-linked intracellular pathogens [8-10].

Prenatal maternal infections, such as influenza, rubella, Herpes simplex virus (HSV), and measles as well as infection by bacterial pathogens, such as Mycoplasma ssp., Chlamydia pneumoniae, and Borrelia burgdorferi, have been studied and associated with the development of ASD [11-14].

Compared to children with typical development, those with ASD (adjusted odds ratio [aOR] 7.92; 95\% confidence interval [CI] 4.89-12.85) are more likely to have at least 1 frequent gastrointestinal symptom [15]. The communication between gut microbiota and blood-brain barrier (BBB) is initiated during gestation and propagated throughout life and increased BBB permeability is associated with a reduced expression of tight-junction proteins [16].

Immunological anomalies involving cytokines (interleukin [IL]-1R, IL-6, IL-8, IL-10, and IL-17), immunoglobulins, inflammation, and cellular activation have also been noted in individuals with autism [17-19]. Individuals with ASD demonstrate active inflammation in the central nervous system (CNS); a proinflammatory profile in postmortem brains of people diagnosed with ASD indicated elevated cytokines and activated neuroglial responses $[20,21]$, suggesting that future therapies such as manipulating cytokine secretion during pregnancy can prevent the development of abnormal behaviors in offspring [22]. The detection of ASD markers can lead to the identification of more targeted treatments for autism-related symptoms [23].

Considering the burden of the disease, its impact on patients and families, and the already existing research observations, a deeper understanding of the pathophysiological mechanisms of ASD is imperatively needed.

This work offers a detailed up-to-date review of the literature investigating the involvement of intracellular infectious pathogens in ASD. It also generates a putative pathophysiological mechanism that could explain the observed association between infections and ASD.

\section{Methods}

We reviewed and collected studies concerning potential associations between intracellular pathogens like viral, bacterial, and parasite infection and the risk of ASD. To identify relevant studies, we searched the PubMed, EMBASE, SCOPUS, and ISI Web of Science databases. Keywords utilized included "autism," "autistic," "ASD," "Asperger' $s$ " in combinations with the terms "intracellular pathogens," "bacteria," "Borrelia burgdorferi," "viruses," "Chlamydia sp.," "Mycoplasma ssp.," "Bartonella," "HHV6," "bacteria" and "Toxoplasma gondii." The reference lists from identified studies were also hand-searched for any additional studies. Review articles, hypothesis papers, and Letters to the Editor that did not present unique or new data were excluded. This review included original articles in humans related to case-controls, cohorts, and cross-sectional studies. Software IntAct View v4.1.4 was used to design the IL-6 interactome.

\section{Results}

For this review, 14 publications were included; 9 casecontrol studies (Table 1), 2 cohorts (Table 2), and 3 crosssectional studies (Table 3 ) were analyzed, as described below.

\section{Infections during Pregnancy}

To examine the association between ASD and infections, 1 case-control study was performed using data and blood samples from the Danish historic birth cohort 
Table 1. Case-control studies

\begin{tabular}{|c|c|c|}
\hline Ref. No. & Main aims & Procedure and results \\
\hline [14] & $\begin{array}{l}\text { To measure the prevalence of C. pneumoniae, } \\
\text { Mycoplasma ssp., and Human herpesvirus } 6 \\
\text { (HHV6) }\end{array}$ & $\begin{array}{l}\text { Control subjects }=45 \text {; ASD subjects }=45 \text {. } \\
\text { Blood samples were analyzed by PCR. } \\
\text { Mycoplasma ssp.: ASD } 58.3 \% \text {, controls } 4.7 \%, p<0.001 \text {. } \\
\text { C. pneumoniae: ASD } 8.3 \% \text {, controls } 2.1 \%, p<0.01 \text {. } \\
\text { HHV6: ASD } 29.2 \% \text {, controls } 8.3 \%, p<0.01 \text {; no differences in the prevalence of infections } \\
\text { were found between urban and rural or between male and females subjects. }\end{array}$ \\
\hline
\end{tabular}

[29] To evaluate the presence of Cytomegalovirus (CMV), Epstein-Barr virus (EBV), Herpes simplex virus types 1 and 2 (HSV1, HSV2), HHV6, BK virus (BKV), JC virus (JCV), and Simian virus 40 (SV40)

\section{Control subjects $=13 ;$ ASD subjects $=15$.}

Postmortem temporocortical tissue was analyzed by PCR.

Viral genome: ASD $80 \%$, controls $62 \%$ (OR 2.5, $p=0.40)$.

BKV, JCV, and SV40 either singly or in combination: ASD 67\%, controls 23\% (OR 6.67, $p=0.029)$.

All other viruses presented no differences between groups.

Polyviral infections did not differ between groups (OR 6.67, $p=0.084$ ).

Polyomaviruses represent the only viral species significantly more present in the postmortem brains of ASD patients (67\%), $p<0.05$.

\section{[33] To test IgG, IgM, and IgA antibodies against 9 different neuron-specific antigens, milk and bacterial peptides, including C. pneumoniae}

Control subjects $=40$, ASD subjects $=40$.

Blood samples were used, and the antigens were analyzed by ELISA. All antigens tested were significantly increased in ASD, $p<0.001$.

[24] To investigate the association between infection and ASD during 4 pre-defined time periods utilizing a Danish historic birth cohort (HBC)

\section{Control subjects $=820$, ASD subjects $=414$.}

This study used blood samples from HBC and data from Danish nation-wide health registers; the prevalence of diagnosed infections was higher in the ASD cases during all 4 follow-up periods.

ASD was associated with a higher risk of hospital admittance due to infection during the first year of life $(p=0.02)$ and at the end of the follow-up period $(p=0.03)$.

Bacterial infections at the end of the follow-up period were slightly less prevalent in ASD cases than in controls (OR 0.84, $p=0.31$ ).

[30] To compare the exposure rate and titer of antibodies to Varicella zoster virus (VZV)

Control subjects $=46$, ASD subjects $=54$.

The exposure rate and titer of anti-VZV antibodies were significantly higher in children with ASD compared to controls ( 59 vs. $39 \%$ and 694 vs. $94 \mathrm{mIU} / \mathrm{mL}$, respectively, $p=$ 0.045).

[25] To investigate the association between infections in the first 2 years of life and a subsequent diagnosis of ASD

Control subjects $=2,100$, ASD subjects $=403$.

A significant case-control difference occurred in the first 30 days of life, during which time a significantly greater proportion of children later identified with ASD were diagnosed with an infection (22.6 vs. $18.7 \%$; $p=0.03$; aOR 1.4 ; $95 \%$ CI $1.1-1.7$ ).

[31] To evaluate the seropositivity rate and antibody level of HSV1 and HSV2 in children with ASD

Control subjects $=46$, ASD subjects $=54$.

Seropositivity rate and levels of anti-HSV1/2 were similar in cases and controls; exposure vs. same-aged healthy controls to HSV2 was minimal.

[32] To evaluate seropositivity rate and levels of antibodies to HHV6 and HHV8 in children with ASD vs. controls

(HBC) [24]. They analyzed 4 predefined time periods to compare groups: (1) infections during pregnancy; (2) infections during the first month after birth; (3) infections during the first year after birth; (4) infections until the end of follow-up. The infections were examined in 2 subcategories: viral and bacterial. The study population included 414 ASD cases and 820 controls matched for gender and year of birth. The prevalence of infections was 
Table 2. Cohort studies

\begin{tabular}{lll}
\hline Ref. No. & Main aims & Procedure and results \\
\hline$[11]$ & $\begin{array}{l}\text { To evaluate the association between } \\
\text { hospitalization for infection in the } \\
\text { perinatal period or childhood and ASD }\end{array}$ & $\begin{array}{l}\text { ASD subjects: } 7,379 \text { children (total 1,418,152). } \\
\text { Children admitted to hospital for any infectious disease displayed an } \\
\text { increased rate of ASD diagnoses, this association was found to be similar for } \\
\text { infectious disease of bacterial and viral origin (HR 1.38). } \\
\text { Children admitted to the hospital for noninfectious disease also displayed an } \\
\text { increase rate of ASD diagnoses (HR 1.76). }\end{array}$ \\
$\begin{array}{ll}\text { To evaluate the prevalence of common } \\
\text { infections, febrile episodes, and use of } \\
\text { antibiotics reported by the mother } \\
\text { during pregnancy, and the risk for ASD } \\
\text { and infantile autism in the offspring }\end{array}$ & $\begin{array}{l}\text { ASD subjects: 976 (from a total of 96,736). } \\
\text { Conducted by self-reported telephone interview; maternal influenza } \\
\text { infection was associated with a 2-fold increased risk of infantile autism, } \\
\text { prolonged episodes of fever caused a 3-fold increased risk of infantile autism, } \\
\text { and the use of various antibiotics during pregnancy was a potential risk } \\
\text { factor for ASD/infantile autism. } \\
\text { No association was described between specific types of self-reported } \\
\text { common infection during pregnancy and ASD in the offspring, and there } \\
\text { was no increased risk for ASD after common infections in specific } \\
\text { trimesters. }\end{array}$ \\
&
\end{tabular}

Table 3. Cross-sectional studies

\begin{tabular}{|c|c|c|}
\hline$[14]$ & $\begin{array}{l}\text { Analyze the prevalence of chronic } \\
\text { infections }\end{array}$ & $\begin{array}{l}\text { ASD subjects }=48 \text {. } \\
\text { Blood samples were evaluated by PCR. } \\
\text { Mycoplasma ssp. } 50-60 \% \text {, HHV6 }<30 \% \text {, C. pneumoniae }<10 \% \text {, B. burgdorferi } \\
20-30 \% \text {, S. aureus }<2 \% \text {, and hepatitis virus }<2 \% \text {. }\end{array}$ \\
\hline$[20]$ & $\begin{array}{l}\text { Investigate whether immune-mediated } \\
\text { mechanisms are involved in the } \\
\text { pathogenesis of autism }\end{array}$ & $\begin{array}{l}\text { ASD subjects = } 11 . \\
\text { Brain tissues and cerebrospinal fluid were analyzed by } \\
\text { immunocytochemistry. } \\
\text { Active neuroinflammatory processes in the cerebral cortex, white matter, } \\
\text { and cerebellum of ASD patients. }\end{array}$ \\
\hline$[28]$ & Evaluate the prevalence of $B$. burgdorferi & $\begin{array}{l}\text { Control subjects }=55 \text {; ASD subjects }=104 \text {. } \\
\text { Blood samples were used, and antigens were analyzed by quantitative } \\
\text { luciferase immunoprecipitation system (LIPS). } \\
\text { No evidence of B. burgdorferi infection was detected in children with or } \\
\text { without ASD. }\end{array}$ \\
\hline
\end{tabular}

higher in the ASD cases during all follow-up periods, but significantly higher during the last 2 follow-up periods. ASD was associated with a higher risk of hospital admittance due to infection during the first year of life (OR 1.48, $p=0.02$ ), and at the end of follow-up (OR 1.3, $p=0.03$ ). Bacterial infections at the end of follow-up were slightly less prevalent in the ASD cases than in the controls (OR $0.84, p=0.31$ ).

With a similar purpose, another case-control study was performed on children born at Kaiser Permanente, Oakland, CA, USA, from 1995 to 1999 (403 ASD cases and 2,100 controls were included) [25]. General infections diagnosed in the first 2 years of life were recorded slightly less often in children with autism than in control children (95.0 vs. $97.5 \%$ ) but this was not significant. However, a case-control difference occurred in the first 30 days of life, during which time a significantly greater proportion of children later identified with ASD were diagnosed with an infection (22.6 vs. $18.7 \%$; $p=0.03$; aOR 1.4 [95\% CI 1.1-1.7]).

To investigate the association between hospitalization for infection in the peri-/neonatal period or childhood 
and a diagnosis of ASD, 2 population-based cohort studies from Denmark were included in this review. The first aimed to investigate the association between hospitalization for infection in the peri-/neonatal period or childhood and the diagnosis of ASD [11]. A total of 7,379 children were diagnosed as having ASD. Children admitted to the hospital for any infectious disease displayed an increased rate of ASD diagnoses. This association was found to be similar for infectious diseases with a bacterial or viral origin (hazard ratio [HR] 1.38; 95\% CI 1.31-1.45). Children admitted to the hospital for noninfectious disease also displayed an increased rate of ASD diagnosis (HR 1.76; 95\% CI 1.68-1.86). The results did not suggest causality because a general association was observed across different infection groups. The second study by the same research group investigated the occurrence of common infections, febrile episodes, the use of antibiotics by the mother during pregnancy, and the risk of ASD [26]. A total of 976 ASD children (1\% of the cohort) were analyzed. The results suggest that maternal influenza infection was associated with a 2 -fold increased risk of infantile autism, a prolonged episode of fever caused a 3 -fold increased risk of infantile autism, and the use of various antibiotics during pregnancy was a potential risk factor for ASD/infantile autism, but the authors emphasize that these data do not suggest a strong risk factor. They observed no association between specific types of self-reported common infection during pregnancy and ASD in the offspring, and no increased risk for ASD after common infections during specific trimesters.

\section{Evidence of Infection with Intracellular Pathogens \\ Found in ASD Patients}

One of the case-control studies was conducted in California, USA, and examined the blood of 48 ASD patients and 45 age-matched controls [14]. It showed an important difference in levels of C. pneumoniae (OR 5.6, $p<$ 0.001), Mycoplasma ssp. (OR 13.8, $p<0.01$ ) and Human herpesvirus (HHV)6 (OR 4.5, $p<0.01$ ).

Another study, which analyzed the blood serum of 48 ASD children compared to 34 controls, presented no significant differences between groups: $85 \%$ of ASD children and $82 \%$ of controls had positive titers of measles antibodies ( $p=0.201$ ), and $76 \%$ of ASD children versus $61 \%$ of controls had positive titers of HHV6 antibody ( $p=$ 0.128 ) [27]. However, the study showed an association between serology and brain autoantibodies in ASD samples, while all controls were negative for these antibodies.

A retrospective, cross-sectional cohort was used to assess the frequency of $B$. burgdorferi seropositivity in

Intracellular Pathogen Infections and Immune Response in Autism young children with or without ASD from the East Coast and the Midwest, USA [28]. The serum samples were tested using a quantitative luciferase immunoprecipitation system for detecting B. burgdorferi antibodies. A total of 104 ASD subjects, 24 developmentally delayed, and 55 typically developing children were analyzed. The antibody levels in all children were below the cut-off value of $10,000 \mathrm{LU}$, indicating no evidence of $B$. burgdorferi infection in children with and without ASD.

To assess the presence of several neurotropic viruses, including HSV1, HSV2, and HHV6, 1 study examined postmortem brains of 15 ASD patients and 13 controls, using nested polymerase chain reaction (PCR) followed by DNA sequence analysis. Polyomaviruses represented the only viral species significantly more presented in the tissues of ASD subjects than controls (67 vs. $23 \%, p<$ $0.05)$. However, HSV1, HSV2, and HHV6 were found in $\leq 3$ cases and/or controls, with no differences in incidence [29].

Three studies by the same group from Italy were included in this review. They compared the prevalence and the titer of anti-Varicella zoster virus (VZV) antibodies in 54 ASD children and 35 controls [30]. The mean age was $6.1( \pm 2.5)$ years for cases and $5.9( \pm 2.8)$ years for controls. They found that exposure rate and titer of anti-VZV antibodies were significantly higher in children with ASD cases than in controls (59 vs. $39 \%$ and 694 vs. $94 \mathrm{mlU} / \mathrm{mL}$, respectively, $p=0.045)$. In another study, they compared the seropositivity rate and levels of IgG antibodies to HSV1 and HSV2 in blood samples from 54 ASD patients and 46 controls, using an indirect chemioluminescence immunoassay [31]. The mean age was $6.1( \pm 2.5)$ years for cases and $5.9( \pm 2.8)$ years for controls. They reported a similar prevalence in the ASD and control groups (total HSV, 29 vs. $21.7 \%$; HSV2, 1.9 vs. $0 \%$, respectively, $p=$ $0.775)$. They also studied the seropositivity rate and levels of antibodies to HHV6 and HHV8 in 30 ASD children and 28 control individuals. The median age was 5.83 years for cases and 5.88 for controls. No significative differences were found in the seropositivity rate and levels of antibodies ( $p=0.798)$ to HHV6 and HHV8 [32].

An immunological examination was performed on the blood samples of 40 ASD patients (3-12 years of age) and 40 age- and sex-matched controls. Enzyme-linked immunosorbent assay (ELISA) was used for testing IgG, IgM, and IgA antibodies against 9 different neuron-specific antigens, milk and bacterial peptides, including C. pneumoniae, in the sera of subjects. For all antigens tested, the differences between control and ASD were highly significant $(p<0.001)$, suggesting that these antibodies may 
cross the $\mathrm{BBB}$ and combine with brain tissue antigens forming immune complexes, thus further damaging the neurological tissue [33].

In 2007, a cross-sectional study was conducted to investigate the prevalence of chronic infections in 48 ASD patients. They showed the following prevalence rates: Mycoplasma ssp. 50-60\%, HHV6 <30\%, C. pneumoniae $<10 \%$, B. burgdorferi 20-30\%, Staphylococcus aureus $<2 \%$, and hepatitis virus $<2 \%[14]$.

To investigate whether immune-mediated mechanisms are involved in the pathogenesis of autism, the brain tissue and cerebrospinal fluid of 11 ASD patients were analyzed by immunocytochemistry, and active neuroinflammatory processes in the cerebral cortex, white matter, and cerebellum of autistic patients were identified [20].

No clinical studies have specifically linked autism to toxoplasmosis, but there is biological evidence of an association between this infection and the risk of ASD [34]. A link with toxoplasmosis has been relatively well explored in psychiatric studies, especially in schizophrenia and bipolar disorder $[35,36]$. However, no clinical study has specifically shown a direct connection between ASD and toxoplasmosis and biological evidence of an association between this parasitic infection and the presence of ASD symptoms is scarce.

\section{Discussion}

There are a limited number of published studies on to the association between intracellular pathogens and ASD. We nevertheless found 5 case-control studies that presented an association between infections and ASD [14, $24,25,30,33$.

One of the cohort studies analyzed demonstrated that maternal influenza infection increased ASD risk 2-fold [26] and another showed that children admitted to hospital for any infection displayed an increased rate of ASD diagnosis [11]. The cross-sectional studies demonstrated that ASD patients presented many chronical infections [14] and active neuroinflammatory processes in the cerebral cortex, white matter, and cerebellum [20].

Variability/controversy regarding results might be explained by small sample groups in some studies, heterogeneous methods, and the design of study protocols. To address the high phenotypic heterogeneity, 1 study suggested a division into clinical subgroups based on symptoms clusters: (1) circadian rhythm abnormalities, (2) circadian rhythm and immunological problems, and (3) ste- reotypical behaviors, (4) mixed with some more prominent neurodevelopmental abnormalities [37].

However, all the studies analyzed indicate the inflammatory process as a common key point in response to the infections $[8,20,33,38]$. The potential role of maternal infections or inflammation during pregnancy in the etiology of autism has been previously reported $[8,11,38,39]$. The $\mathrm{BBB}$ is permeable during fetal development and can be compromised by infections. Individuals with ASD present with increased proinflammatory cytokines in the brain as well as the activation of resident immune cells, such as microglia. Antinuclear antibodies in targeted brain tissues have been described in both children with autism and their mothers [40].

Proinflammatory cytokines such as IL- $1 \beta$, IL-6, and tumor necrosis factor (TNF)- $\alpha$ are essential to the inflammatory response; they mediate febrile reactions, activate phagocytic cells, increase vascular permeability, and promote the release of plasma-derived inflammatory mediators [41]. Increased levels of the cytokine IL- 6 are suggested to be associated with ASD [42]. An imbalance in the production of inflammatory (IL- $1 \beta$ and IL- 6 ) and contraregulatory (IL-10) cytokines in the monocytes of ASD patients was recently described [43].

The maternal immune activation (MIA) system raises the level of IL-6, which can bind to gp130, leading to JAKSTAT3 activation, acute-phase protein expression, and downregulation of placental growth hormone production. This signaling cascade reduces insulin-like grow factor. These various changes in endocrine factors likely cause acute placental pathophysiology with subsequent effects on fetal development [8]. The rapid inflammatory responses are mediated via pattern recognition receptors (PRR), expressed particularly by microglia/macrophages. PRR sense pathogen-associated molecules from microorganisms or damage-associated molecular pattern molecules that are released from injured tissue, and activate the intracellular signaling pathways that lead to an expression of inflammatory mediators (cytokines and chemokines) in the CNS and periphery. The most common type of PRR identified in the CNS are toll-like receptors (TLR) [44].

A recent review suggests that inflammation in utero can lead to preterm labor and has also been strongly associated with adverse neurodevelopmental outcomes. Premature babies have an approximately 4-fold higher risk of developing ASD and are also more vulnerable to infection. Perinatal mast cell activation by infectious, stress-related, environmental, or allergic triggers can lead to the release of proinflammatory and neurotoxic mole- 
cules, thereby contributing to brain inflammation, at least in a subgroup of ASD patients [45].

Dietary peptides, bacterial toxins, and xenobiotics bind to lymphocyte receptors and/or tissue enzymes, resulting in autoimmune reactions in children with autism [46]. Bacterial LPS activates TLR- 4 on mast cells and induces the selective release of TNF. Mast cells also express viral TLR3 , the activation of which by viral double-stranded RNA induces the release of IL- 6 and TNF. Elevated IL- 6 gene expression has been noted in the brain of autistic patients. In contrast, the TGF- $\beta 1$ level is low in ASD patients; this inhibits mast cell function. Mast cell-derived IL-9 induces intestinal permeability and predisposes to oral antigen hypersensitivity in children. It also exacerbates brain toxic lesions in offspring. When mast cells are activated, this may cause a disruption in the BBB via the cytokines, permit neurotoxic molecules to enter the brain, and result in brain inflammation, contributing to ASD pathogenesis [45].

An experimental study suggested MIA alters fetal brain development through IL-6. In a mouse model, a single maternal injection of IL-6 during pregnancy caused prepulse inhibition and latent inhibition deficits in the adult offspring. IL-6 antibody, that inactivates IL-6, improves autism-like behaviors and normalizes the associated changes in gene expression in the brains of adult offspring [47].

The precise mechanisms whereby latent or persistent inflammation negatively affects the neurobiological and neurochemical abnormalities relevant to autism are currently the subject of extensive experimental studies. The further elucidation of such mechanisms may lead to the establishment of novel immunomodulatory interventions that could help prevent abnormal brain development and long-term mental illness in subjects with prenatal infectious/inflammatory histories [41].

IL-6 is a multifunctional cytokine that plays a key role in biological processes, e.g., the inflammatory response to acute and chronic infections, making it a promising candidate for clinical treatment. HHV8, also known as Kaposi sarcoma-associated herpesvirus, encodes a molecule similar to IL-6 called viral IL-6 (VIL-6). VIL-6 is reported to directly bind to gp130 and transmit signals in the absence of IL-6R, and it is widely accepted that it plays a significant role in the pathology of HHV8-associated diseases such as Castleman disease [48]. Related to the interleukin profile, the use of anti-IL-6R therapy could indicate a promising tool to modulate IL-6 signaling and production for treating inflammatory diseases.

IL-6 elevation in the brain promotes an inflammatory response that could be involved in the mediation of au-

Intracellular Pathogen Infections and Immune Response in Autism

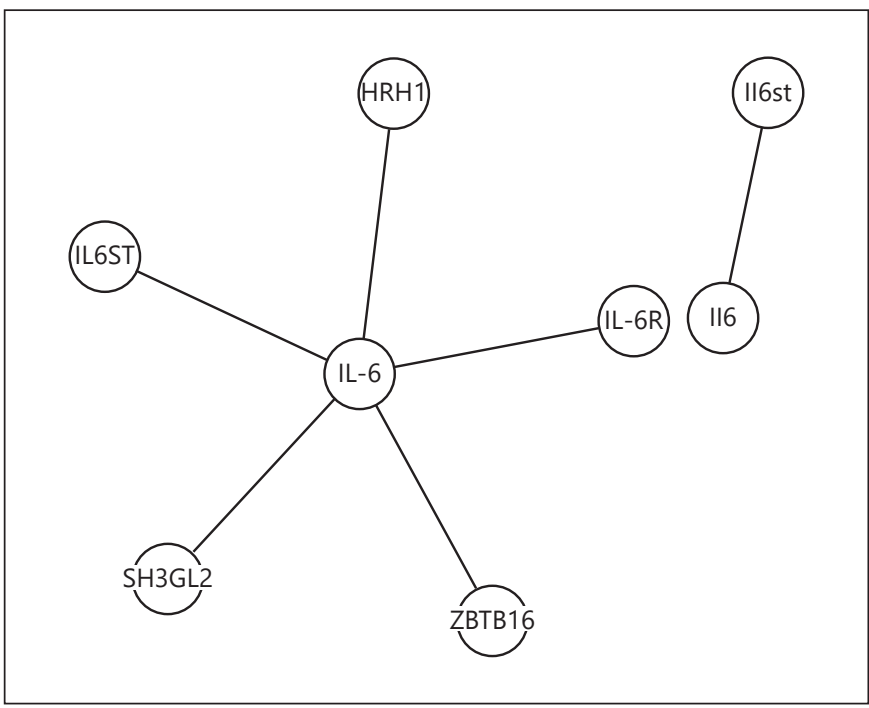

Fig. 1. Interactome for interleukin 6. IL-6, interleukin 6; IL-6R, IL-6 receptor; ZBTB16, zinc finger and BTB domain containing 16; SH3GL2, SH3-domain GRB2-like 2; IL6ST, IL-6 signal transducer; HRH1, histamine receptor $\mathrm{H} 1$.

tism behaviors by impairment of neuroanatomical structures and neuronal plasticity [49]. The neuroimmune network includes astrocytes and microglia, immune mediators, and other classical immune pathways that play an important role in brain development, and is critical for the process of neuronal migration, axonal growth, neuronal positioning, cortical lamination, as well as dendritic and synapse formation. In this context, defects in neuroimmune factors could be involved in neuropsychiatric disorders [50].

Figure 1 indicates the IL- 6 interactome, pointing out important molecules to be further investigated, including IL-6, IL-6R, zinc finger and BTB domain containing 16 (ZBTB16), SH3-domain GRB2-like 2 (SH3GL2), IL-6 signal transducer (IL6ST) and histamine receptor H1 (HRH1). Ligands of the histamine receptor are considered potential therapeutic agents for the treatment of different brain disorders and cognitive impairments, like autism [51]. Among these possible targets described, HRH1 has been associated with the diagnosis of autism in recent research [52] and ZBTB16 was associated with depression [53]. Based on the this review, IL-6 appears to be a key player in the inflammatory process in ASD.

It is important to bear in mind some limitations of this study. First, most of the studies included in this review did not present extensively variable control for many covariates, which raise the concern of residual confounding by 
unmeasured factors. Second, elevated levels of proinflammatory markers (e.g., IL-6) may not be specific to childhood autism among depressive disorders, since significant levels of IL-5 and IL- 6 have been detected in other childhood psychiatric disorders [54]. Furthermore, the use of serotonin reuptake inhibitors, which have been associated with autism when taken during pregnancy [55], was not associated with increased levels of proinflammatory markers [56].

In summary, studies including less investigated pathogens like Brucella sp. and Streptococcus sp. are necessary to investigate the prevalence of infections in ASD patients and their mothers during pregnancy. In addition, specific protocols need to be developed to routinely screen infections with the aim of developing preventive strategies and/or more effective treatments.

\section{Concluding Remarks}

The immune responses generated by the organisms that cause perinatal maternal infection, i.e., bacteria, viruses, or parasites, have been associated with the develop- ment of autism in offspring. The molecular mechanisms involved in the physiological and biochemical changes transmitted from the mother during chronic or acute inflammation should be further investigated in order to develop immunomodulatory preventive measures against ASD.

\section{Acknowledgements}

This work was supported by the Institut National de la Santé et de la Recherche Médicale (INSERM), Assistance Publique des Hôpitaux de Paris (AP-HP), Fondation FondaMental (RTRS Santé Mentale), and by the Investissements d'Avenir Program of the Agence Nationale pour la Recherche (ANR-11-IDEX-0004-02). Dr. Dargél is supported by a grant from Fondation FondaMental. Dr. Abib is supported by a grant from Comissão de Aperfeiçoamento e Pesquisa (CAPES), CAPES-COFECUB project 796-14, and Dr. Gottfried by CAPES BEX-1531/14-0.

\section{Disclosure Statement}

There were no conflicts of interest.

\section{References}

1 American Psychiatric Association: Diagnostic and Statistical Manual of Mental Disorders, ed 5 (DSM-5) 2013. DOI: 10.1176/appi. books. 9780890425596.744053

2 Developmental Disabilities Monitoring Network Surveillance Year 2010 Principal Investigators, Centers for Disease Control and Prevention (CDC): Prevalence of autism spectrum disorder among children aged 8 years - autism and developmental disabilities monitoring network, 11 sites, United States, 2010. MMWR Surveill Summ 2014;63:1-21.

3 Chaste P, Leboyer M: Autism risk factors: genes, environment, and gene-environment interactions. Dialogues Clin Neurosci 2012; 14:281-292.

4 Constantino JN, Charman T: Diagnosis of autism spectrum disorder: reconciling the syndrome, its diverse origins, and variation in expression. Lancet Neurol 2016;15:279-291.

5 Torres AR, Sweeten TL, Johnson RC, Odell D, Westover JB, Bray-Ward P, et al: Common genetic variants found in HLA and KIR immune genes in autism spectrum disorder. Front Neurosci 2016;10:463.

6 Bennabi M, Delorme R, Oliveira J, Fortier C, Lajnef M, Boukouaci W, et al: Dectin-1 polymorphism: a genetic disease specifier in autism spectrum disorders? PLoS One 2015; 10:e0137339.
7 Campbell DB, Sutcliffe JS, Ebert PJ, Militerni $\mathrm{R}$, Bravaccio $\mathrm{C}$, Trillo $\mathrm{S}$, et al: A genetic variant that disrupts MET transcription is associated with autism. Proc Natl Acad Sci USA 2006; 103:16834-16839.

8 Patterson PH: Maternal infection and immune involvement in autism. Trends $\mathrm{Mol}$ Med 2011;17:389-394.

9 Patterson PH: Maternal infection and autism. Brain Behav Immun 2012;26:393.

10 Hsiao EY, Patterson PH: Placental regulation of maternal-fetal interactions and brain development. Dev Neurobiol 2012;72:13171326.

11 Atladóttir HÓ, Thorsen P, Schendel DE, Østergaard L, Lemcke S, Parner ET: Association of hospitalization for infection in childhood with diagnosis of autism spectrum disorders. Arch Pediatr Adolesc Med 2010;164: 470-477.

12 Meyer U, Engler A, Weber L, Schedlowski M, Feldon J: Preliminary evidence for a modulation of fetal dopaminergic development by maternal immune activation during pregnancy. Neuroscience 2008;154:701-709.

13 Libbey JE, Sweeten TL, McMahon WM, Fujinami RS: Autistic disorder and viral infections. J Neurovirol 2005;11:1-10.
14 Nicolson GL, Gan R, Nicolson NL, Haier J: Evidence for Mycoplasma ssp., Chlamydia pneunomiae, and Human herpesvirus- 6 coinfections in the blood of patients with autistic spectrum disorders. J Neurosci Res 2007;85: 1143-1148.

15 Chaidez V, Hansen RL, Hertz-Picciotto I: Gastrointestinal problems in children with autism, developmental delays or typical development. J Autism Dev Disord 2014;44: 1117-1127.

16 Braniste V, Al-Asmakh M, Kowal C, Anuar F, Abbaspour A, Tóth $\mathrm{M}$, et al: The gut microbiota influences blood-brain barrier permeability in mice. Sci Transl Med 2014;6:263ra158.

17 Goines P, Van de Water J: The immune system's role in the biology of autism. Curr Opin Neurol 2010;23:111-117.

18 Kugelberg E: Neuroimmunology: IL-17A mediates a path to autism 2016;16:205-205.

19 Krakowiak P, Goines PE, Tancredi DJ, Ashwood P, Hansen RL, Hertz-Picciotto I, et al: Neonatal cytokine profiles associated with autism spectrum disorder. Biol Psychiatry 2017; 81:442-451.

20 Vargas DL, Nascimbene C, Krishnan C, Zimmerman AW, Pardo CA: Neuroglial activation and neuroinflammation in the brain of patients with autism. Ann Neurol 2005;57: 67-81. 
21 Gottfried C, Bambini-Junior V, Francis F, Riesgo R, Savino W: The impact of neuroimmune alterations in autism spectrum disorder. Front Psychiatry 2015;6:121.

22 Careaga M, Water J, Ashwood P: Immune dysfunction in autism: a pathway to treatment. Neurotherapeutics 2010;7:283-292.

23 Masi A, Breen EJ, Alvares GA, Glozier N, Hickie IB, Hunt A, et al: Cytokine levels and associations with symptom severity in male and female children with autism spectrum disorder. Mol Autism 2017;8:63.

24 Abdallah MW, Larsen N, Mortensen EL, Atladóttir HÓ, Nørgaard-Pedersen B, BonefeldJørgensen EC, et al: Neonatal levels of cytokines and risk of autism spectrum disorders: an exploratory register-based historic birth cohort study utilizing the Danish Newborn Screening Biobank. J Neuroimmunol 2012; 252:75-82.

25 Rosen NJ, Yoshida CK, Croen LA: Infection in the first 2 years of life and autism spectrum disorders. Pediatrics 2007;119:e61-e69.

26 Atladóttir HÓ, Henriksen TB, Schendel DE, Parner ET: Autism after infection, febrile episodes, and antibiotic use during pregnancy: an exploratory study. Pediatrics 2012; 130:e1447-e1454.

27 Singh VK, Lin SX, Yang VC: Serological association of measles virus and Human herpesvirus-6 with brain autoantibodies in autism. Clin Immunol Immunopathol 1998;89:105108.

28 Burbelo PD, Swedo SE, Thurm A, Bayat A, Levin AE, Marques A, et al: Lack of serum antibodies against Borrelia burgdorferi in children with autism. Clin Vaccine Immunol 2013;20:1092-1093.

29 Lintas C, Altieri L, Lombardi F, Sacco R, Persico AM: Association of autism with Polyomavirus infection in postmortem brains. J Neurovirol 2010;16:141-149.

30 Gentile I, Zappulo E, Bonavolta R, Maresca R, Riccio MP, Buonomo AR, et al: Exposure to Varicella zoster virus is higher in children with autism spectrum disorder than in healthy controls. Results from a case-control study. In Vivo 2014;28:627-631.

31 Gentile I, Zappulo E, Bonavolta R, Maresca R, Riccio MP, Buonomo AR, et al: Prevalence of herpes simplex virus 1 and 2 antibodies in patients with autism spectrum disorders. In Vivo 2014:28:667-671.

32 Gentile I, Zappulo E, Coppola N, Bonavolta R, Portella G, Cernia DS, et al: Prevalence of HHV-6 and HHV-8 antibodies in patients with autism spectrum disorders. In Vivo 2013;27:843-849.
33 Vojdani A, Campbell AW, Anyanwu E, Kashanian A, Bock K, Vojdani E: Antibodies to neuron-specific antigens in children with autism: possible cross-reaction with encephalitogenic proteins from milk, Chlamydia pneumoniae and Streptococcus group A. J Neuroimmunol 2002;129:168-177.

34 Carter CJ: Toxoplasmosis and polygenic disease susceptibility genes: extensive Toxoplasma gondii host/pathogen interactome enrichment in nine psychiatric or neurological disorders. J Pathog 2013;2013:1-29.

35 Sutterland AL, Fond G, Kuin A, Koeter MWJ, Lutter R, van Gool T, et al: Beyond the association. Toxoplasma gondii in schizophrenia, bipolar disorder, and addiction: systematic review and meta-analysis. Acta Psychiatr Scand 2015;132:161-179.

36 Fond G, Boyer L, Gaman A, Laouamri H, At tiba D, Richard J-R, et al: Treatment with anti-toxoplasmic activity (TATA) for toxoplasma positive patients with bipolar disorders or schizophrenia: a cross-sectional study. J Psychiatr Res 2015;63:58-64.

37 Sacco R, Militerni R, Frolli A, Bravaccio C, Gritti A, Elia M, et al: Clinical, morphological, and biochemical correlates of head circumference in autism. Biol Psychiatry 2007;62:10381047.

38 Brown AS, Sourander A, Hinkka-Yli-Salomäki S, McKeague IW, Sundvall J, Surcel H-M: Elevated maternal C-reactive protein and autism in a national birth cohort. Mol Psychiatry 2014;19:259-264.

39 Rossignol DA, Genuis SJ, Frye RE: Environmental toxicants and autism spectrum disorders: a systematic review. Transl Psychiatry 2014;4:e360-e360.

40 Goines P, Van de Water J: The immune system's role in the biology of autism. Curr Opin Neurol 2010;23:111-117.

41 Meyer U, Feldon J, Dammann O: Schizophrenia and autism: both shared and disorderspecific pathogenesis via perinatal inflammation? Pediatr Res 2011;69:26R-33R.

42 Bransfield RC, Wulfman JS, Harvey WT, Usman AI: The association between tick-borne infections, Lyme borreliosis and autism spectrum disorders. Med Hypotheses 2008;70: 967-974.

43 Jyonouchi H, Geng L, Davidow AL: Cytokine profiles by peripheral blood monocytes are associated with changes in behavioral symptoms following immune insults in a subset of ASD subjects: an inflammatory subtype? J Neuroinflammation 2014;11:187.

44 Hagberg H, Gressens P, Mallard C: Inflammation during fetal and neonatal life: Implications for neurologic and neuropsychiatric disease in children and adults. Ann Neurol 2012;71:444-457.
45 Theoharides TC, Angelidou A, Alysandratos K-D, Zhang B, Asadi S, Francis K, et al: Mast cell activation and autism. Biochim Biophys Acta 2012;1822:34-41.

46 Vojdani A, O’Bryan T, Green JAA, Mccandless J, Woeller KNN, Vojdani E, et al: Immune response to dietary proteins, gliadin and cerebellar peptides in children with autism. Nutr Neurosci 2004;7:151-161.

47 Smith SEP, Li J, Garbett K, Mirnics K, Patterson $\mathrm{PH}$ : Maternal immune activation alters fetal brain development through interleukin-6. J Neurosci 2007;27:10695-10702.

48 Suthaus J, Stuhlmann-Laeisz C, Tompkins VS, Rosean TR, Klapper W, Tosato G, et al: HHV-8-encoded viral IL-6 collaborates with mouse IL- 6 in the development of multicentric Castleman disease in mice. Blood 2012; 119:5173-5181.

49 Wei H, Alberts I, Li X: Brain IL-6 and autism. Neuroscience 2013;252:320-325.

50 Onore C, Careaga M, Ashwood P: The role of immune dysfunction in the pathophysiology of autism. Brain Behav Immun 2012;26:383392

51 Baronio D, Castro K, Gonchoroski T, de Melo GM, Nunes GDF, Bambini-Junior V, et al: Effects of an H3R antagonist on the animal model of autism induced by prenatal exposure to valproic acid. PLoS One 2015; 10:e0116363.

52 Wright C, Shin JH, Rajpurohit A, Deep-Soboslay A, Collado-Torres L, Brandon NJ, et al: Altered expression of histamine signaling genes in autism spectrum disorder. Transl Psychiatry 2017;7:e1126.

53 Spijker S, Van Zanten JS, De Jong S, Penninx BWJH, van Dyck R, Zitman FG, et al: Stimulated gene expression profiles as a blood marker of major depressive disorder. Biol Psychiatry 2010;68:179-186.

54 Abdallah MW, Hougaard DM, NørgaardPedersen B, Grove J, Bonefeld-Jørgensen EC, Mortensen EL: Infections during pregnancy and after birth, and the risk of autism spectrum disorders: a register-based study utilizing a Danish historic birth cohort. Turk Psikiyatri Derg 2012;23:229-235.

55 Croen LA, Grether JK, Yoshida CK, Odouli R, Hendrick V: Antidepressant use during pregnancy and childhood autism spectrum disorders. Arch Gen Psychiatry 2011;68:1104.

56 Hamer M, Batty G, Marmot MG, Singh-Manoux A, Kivimäki M: Anti-depressant medication use and $\mathrm{C}$-reactive protein: results from two population-based studies. Brain Behav Immun 2011;25:168-173.
Intracellular Pathogen Infections and Immune Response in Autism
Neuroimmunomodulation 2018:25:271-279 